\title{
On the "spin connection foam" picture of quantum gravity from precanonical quantization
}

\author{
Igor V. Kanatchikov* \\ School of Physics and Astronomy, University of St Andrews, \\ St Andrews KY16 9SS, Scotland \\ *E-mail: ik25@st-andrews.ac.uk
}

\begin{abstract}
Precanonical quantization is based on a generalization of the Hamiltonian formalism to field theory, the so-called De Donder-Weyl (DW) theory, which does not require a spacetime splitting and treats the space-time variables on an equal footing. Quantum dynamics is described by a precanonical wave function on the finite dimensional space of field coordinates and space-time coordinates, which satisfies a partial derivative precanonical Schrödinger equation. The standard QFT in the functional Schrödinger representation can be derived from the precanonical quantization in a limiting case. An analysis of the constraints within the DW Hamiltonian formulation of the Einstein-Palatini vielbein formulation of GR and quantization of the generalized Dirac brackets defined on differential forms leads to the covariant precanonical Schrödinger equation for quantum gravity. The resulting dynamics of quantum gravity is described by the wave function or transition amplitudes on the total space of the bundle of spin connections. Thus, precanonical quantization leads to the "spin connection foam" picture of quantum geometry represented by a generally non-Gaussian random field of spin connection coefficients, whose probability distribution is given by the precanonical wave function. The normalizability of precanonical wave functions is argued to lead to the quantum-gravitational avoidance of curvature singularities. Possible connections with LQG are briefly discussed.
\end{abstract}

Keywords: Quantum gravity; precanonical quantization; De Donder-Weyl theory; vielbein gravity; Gerstenhaber algebra; Dirac brackets; Clifford algebra; Lévy processes.

\section{Precanonical quantization of fields}

Contemporary quantum field theory originates from the canonical quantization which is based on the canonical Hamiltonian formalism. The latter dictates a picture of fields as infinite dimensional Hamiltonian systems. It also restricts the consideration to the globally hyperbolic space-times, as it implies a different role of the time dimension, along which the evolution proceeds, and the space dimensions, which label the continuum of degrees of freedom of fields. Many problems we encounter in quantum gravity theories can be traced back to this very origin of QFT.

However, the canonical Hamiltonian formalism is not the only possibility to extend the Hamiltonian formalism from mechanics to field theory. The alternative "Hamiltonizations" (i.e. writing the field equations in the first order form using some generalization of the Legendre transform) known in the calculus of variations $\frac{1}{\underline{1}}$ are, in fact, inherently more geometrical than the canonical formalism, and they treat the space and time variables (i.e. the independent variables of the multiple integral variational problem) on the equal footing, i.e. essentially as multidimensional generalizations of the one-dimensional time parameter in mechanics.

In a sense, those Hamiltonizations of field theories are intermediate between the Lagrangean description and the canonical Hamiltonian formalism. Besides, in the 
case of mechanics, i.e. one-parameter variational problems, all those formulations reduce to the canonical Hamiltonian formalism in mechanics. For this reason we name those formulations "precanonical" and the resulting procedure of quantization of fields "precanonical quantization".

The simplest precanonical formulation is the so-called De Donder-Weyl (DW) theory: $\underline{1}^{-}$for a Lagrangian density $L=L\left(\phi^{a}, \phi_{\mu}^{a}, x^{\nu}\right)$, which is a function of the field variables $\phi^{a}$, their first space-time derivatives $\phi_{\mu}^{a}$, and the space-time variables $x^{\mu}$, one defines the polymomenta $p_{a}^{\mu}:=\frac{\partial L}{\partial \phi_{\mu}^{a}}$ and the DW Hamiltonian function $H\left(\phi^{a}, p_{a}^{\mu}, x^{\mu}\right):=\phi_{\mu}^{a}(\phi, p) p_{a}^{\mu}-L$. Then, if the Legendre transform $\phi_{\nu}^{a} \rightarrow p_{a}^{\nu}$ is regular, the field equations can be cast into the DW Hamiltonian form:

$$
\partial_{\mu} \phi^{a}(x)=\frac{\partial H}{\partial p_{a}^{\mu}}, \quad \partial_{\mu} p_{a}^{\mu}(x)=-\frac{\partial H}{\partial \phi^{a}}
$$

This formulation requires neither a splitting into the space and time nor infinitedimensional spaces of field configurations. The analogue of the extended configuration space here is a finite dimensional space of field variables $\phi^{a}$ and space-time variables $x^{\mu}$, and the analogue of the extended phase space is a finite dimensional space of variables $p_{a}^{\mu}, \phi^{a}$ and $x^{\mu}$. Those spaces are bundles over space-time (see e.g. Ref. 4,5$)$ whose sections are classical field configurations.

While the usual approaches to field quantization work on the infinite dimensional spaces of sections of the above bundles ("the spaces of solutions"), our precanonical quantization approach tries to find a formulation of quantum dynamics of fields in terms of the objects on the finite dimensional bundles themselves, without referring to the remnants of the classical thinking about fields as "field configurations", similarly to the quantum mechanics, which has abandoned the notion of particles' trajectories in favor of the probabilistic concept of their location at different points of the configuration space or propagation amplitudes on this space.

The Poisson brackets in the DW Hamiltonian formulation of field theory in $n$ space-time dimensions are constructed $\underline{\underline{2}}$ using the polysymplectic $(n+1)$-form on the extended polymomentum phase space as the fundamental underlying structure generalizing the symplectic $2-$ form of the canonical Hamiltonian formalism: $\Omega:=d p_{a}^{\mu} \wedge d \phi^{a} \wedge \varpi_{\mu}$, where $\varpi_{\mu}:=\imath_{\partial_{\mu}} \varpi, \varpi:=d x^{0} \wedge \ldots \wedge d x^{n-1}$. The Poisson brackets are defined on differential forms representing the local dynamical variables (or "observables") and they lead to a Gerstenhaber algebra structure, which generalizes the standard Poisson algebra to the DW Hamiltonian formulation of field theory. $\underline{\underline{2}}$

Precanonical quantization of fields $\underline{\underline{3}}$ is based on quantization of the PoissonGerstenhaber brackets of forms ${ }^{2}$ in the DW Hamiltonian theory according to the Dirac quantization rule. It naturally leads to a description of quantum fields in terms of a Clifford (Dirac) algebra valued wave function on the space of field variables and space-time variables, $\Psi\left(\phi^{a}, x^{\mu}\right)$, which fulfills the Dirac-like precanonical Schrödinger equation $\underline{3}$ with the DW Hamiltonian operator $\widehat{H}$ replacing the mass term:

$$
\mathrm{i} \hbar \varkappa \gamma^{\mu} \partial_{\mu} \Psi=\widehat{H} \Psi
$$


where $\varkappa$ is an ultraviolet constant of the dimension of the inverse spatial volume, which originates from the representation of the dimensionful infinitesimal volume elements given by the differential forms $\varpi_{\mu}$ in terms of the dimensionless Dirac matrices $\gamma^{\mu}$, and $\widehat{H}$ is a partial derivative operator with respect to the field variables. The natural appearance of Clifford algebra-valued functions and operators can be argued already on the level of geometric prequantization generalized to the DW Hamiltonian formalism. $\underline{\underline{4}}$ Let us note that the DW Hamiltonian equations (11) can be derived from (2) as the equations on the expectation values of the corresponding precanonical quantum operators. $\underline{\underline{6}}$ The explicit form of a generalization of equation (2) in quantum gravity will be presented below. A validity of the Ehrenfest theorem in this formulation of quantum gravity is to be discussed elsewhere. $\underline{\underline{7}}$

A relation of the above description to the standard QFT in the functional Schrödinger representation has been established in the limit of infinite $\varkappa$ or, more precisely, when $\frac{1}{\varkappa} \gamma^{0} \rightarrow \varpi_{0} . \stackrel{8}{*}$ In this limiting case one can construct the Schrödinger wave functional as the multidimensional Volterra's product integral $\underline{\underline{9}}$ of precanonical wave functions and derive the canonical functional derivative Schrödinger equation from the precanonical Schrödinger equation (2)..$\underline{8}$ Thus the standard QFT turns out to be a singular limiting case of the quantum theory of fields obtained via precanonical quantization. One can view the latter as an "already regularized" quantum field theory in which the UV scale $\varkappa$ is itself an inherent element of the field quantization procedure, that renders standard cutoffs unnecessary.

\section{Precanonical quantization of vielbein gravity and the spin connection foam}

A central role of the Dirac operator in precanonical quantization, which generalizes $i \partial_{t}$ in quantum mechanics (cf. eq. (21)), implies that gravity has to be quantized in vielbein formulation. Here we follow our earlier work $\underline{10}$ (cf. Refs. 11 for an earlier work using the metric formulation).

The Einstein-Palatini Lagrangian density with the cosmological term

$$
\mathfrak{L}=\frac{1}{\kappa} \mathfrak{e} e_{I}^{[\alpha} e_{J}^{\beta]}\left(\partial_{\alpha} \omega_{\beta}^{I J}+\omega_{\alpha}^{I K} \omega_{\beta K}{ }^{J}\right)+\frac{1}{\kappa} \Lambda \mathfrak{e},
$$

where the vielbein components $e_{I}^{\mu}$ and the spin connection coefficients $\omega_{\alpha}^{I J}$ are independent field variables and $\mathfrak{e}:=\left(\operatorname{det}\left\|e_{I}^{\mu}\right\|\right)^{-1}$, leads to a singular DW Hamiltonian theory with the primary constraints

$$
\mathfrak{p}_{e_{I}^{\beta}}^{\alpha}:=\frac{\partial \mathfrak{L}}{\partial \partial_{\alpha} e_{I}^{\beta}} \approx 0, \quad \mathfrak{p}_{\omega_{\beta}^{I J}}^{\alpha}:=\frac{\partial \mathfrak{L}}{\partial \partial_{\alpha} \omega_{\beta}^{I J}} \approx \frac{1}{\kappa} \mathfrak{k} e_{I}^{[\alpha} e_{J}^{\beta]} .
$$

Those are second class, as it follows from the Poisson-Gerstenhaber brackets of $(n-1)$-forms of constraints $\mathfrak{C}_{e_{I}^{\beta}}:=\mathfrak{p}_{e_{I}^{\beta}}^{\alpha} \varpi_{\alpha}$ and $\mathfrak{C}_{\omega_{\beta}^{I J}}:=\left(\mathfrak{p}_{\omega_{\beta}^{I J}}^{\alpha}-\frac{1}{\kappa} \mathfrak{e} e_{I}^{[\alpha} e_{J}^{\beta]}\right) \varpi_{\alpha}$ :

$$
\left\{\mathfrak{C}_{e}, \mathfrak{C}_{e^{\prime}}\right\}=0=\left\{\mathfrak{C}_{\omega}, \mathfrak{C}_{\omega^{\prime}}\right\}, \quad\left\{\mathfrak{C}_{e_{K}^{\gamma}}, \mathfrak{C}_{\omega_{\beta}^{I J}}\right\}=-\frac{1}{\kappa} \partial_{e_{K}^{\gamma}}\left(\mathfrak{e} e_{I}^{[\alpha} e_{J}^{\beta]}\right) \varpi_{\alpha} .
$$


The DW Hamiltonian density obtained from (3) reads

$$
\mathfrak{H}:=\frac{\partial \mathfrak{L}}{\partial \partial_{\alpha} \omega} \mathfrak{p}_{\omega}^{\alpha}+\frac{\partial \mathfrak{L}}{\partial \partial_{\alpha} e} \mathfrak{p}_{e}^{\alpha}-\mathfrak{L}=-\mathfrak{e} e_{I}^{[\alpha} e_{J}^{\beta]} \omega_{\alpha}^{I K} \omega_{\beta K}^{J}-\frac{1}{\kappa} \Lambda \mathfrak{e} .
$$

Using a generalization of the constraints analysis to the DW theory $\underline{\underline{12}}$ we obtain an amazingly simple algebra of the fundamental Dirac brackets on the subalgebra of $(n-1)$ - and 0 -forms: $\underline{10}$

$$
\begin{aligned}
& \left\{\mathfrak{p}_{\omega}^{\alpha} \varpi_{\alpha}, \omega^{\prime} \varpi_{\alpha^{\prime}}\right\}^{\mathrm{D}}=\left\{\mathfrak{p}_{\omega}^{\alpha} \varpi_{\alpha}, \omega^{\prime} \varpi_{\alpha^{\prime}}\right\}=\delta_{\omega}^{\omega^{\prime}} \varpi_{\alpha^{\prime}}, \\
& \left\{\mathfrak{p}_{e}^{\alpha} \varpi_{\alpha}, e^{\prime} \varpi_{\alpha^{\prime}}\right\}^{\mathrm{D}}=0=\left\{\mathfrak{p}_{e}^{\alpha} \varpi_{\alpha}, \mathfrak{p}_{\omega}\right\}^{\mathrm{D}}=\left\{\mathfrak{p}_{e}^{\alpha} \varpi_{\alpha}, \omega^{\prime} \varpi_{\alpha^{\prime}}\right\}^{\mathrm{D}}=\left\{\mathfrak{p}_{\omega}^{\alpha} \varpi_{\alpha}, e^{\prime} \varpi_{\alpha^{\prime}}\right\}^{\mathrm{D}}, \\
& \left\{\mathfrak{p}_{\omega}^{\alpha} \varpi_{\alpha}, \omega^{\prime}\right\}^{\mathrm{D}}=\left\{\mathfrak{p}_{\omega}^{\alpha} \varpi_{\alpha}, \omega^{\prime}\right\}=\delta_{\omega}^{\omega^{\prime}}, \\
& \left\{\mathfrak{p}_{e}^{\alpha} \varpi_{\alpha}, e^{\prime}\right\}^{\mathrm{D}}=0=\left\{\mathfrak{p}_{e}^{\alpha} \varpi_{\alpha}, \mathfrak{p}_{\omega}\right\}^{\mathrm{D}}=\left\{\mathfrak{p}_{e}^{\alpha} \varpi_{\alpha}, \omega\right\}^{\mathrm{D}}=\left\{\mathfrak{p}_{\omega}^{\alpha} \varpi_{\alpha}, e\right\}^{\mathrm{D}}, \\
& \left\{p_{\omega}^{\alpha}, \omega^{\prime} \varpi_{\beta}\right\}^{\mathrm{D}}=\left\{p_{\omega}^{\alpha}, \omega^{\prime} \varpi_{\beta}\right\}=\delta_{\beta}^{\alpha} \delta_{\omega^{\prime}}^{\omega}, \\
& \left\{\mathfrak{p}_{e}^{\alpha}, e^{\prime} \varpi_{\alpha^{\prime}}\right\}^{\mathrm{D}}=0=\left\{\mathfrak{p}_{e}^{\alpha}, \mathfrak{p}_{\omega} \varpi_{\alpha^{\prime}}\right\}^{\mathrm{D}}=\left\{\mathfrak{p}_{e}^{\alpha}, \omega \varpi_{\alpha^{\prime}}\right\}^{\mathrm{D}}=\left\{\mathfrak{p}_{\omega}^{\alpha}, e^{\prime} \varpi_{\alpha^{\prime}}\right\}^{\mathrm{D}}=0 \text {. }
\end{aligned}
$$

The fundamental brackets are quantized according to the generalized Dirac's quantization rule:

$$
[\hat{A}, \hat{B}]=-\mathrm{i} \hbar \mathfrak{e} \widehat{\{A, B}\}^{\mathrm{D}},
$$

in which the presence of the operator $\hat{\mathfrak{e}}$ guarantees that tensor densities are quantized as density-valued operators. Quantization of fundamental Dirac brackets (7)-(12) and the equations of constraints (4) leads to the representation of the operators of vielbeins:

$$
\hat{e}_{I}^{\beta}=-\mathrm{i} \hbar \varkappa \kappa \bar{\gamma}^{J} \frac{\partial}{\partial \omega_{\beta}^{I J}}
$$

and polymomenta of spin connection:

$$
\hat{\mathfrak{p}}_{\omega_{\beta}^{I J}}^{\alpha}=-\hbar^{2} \varkappa^{2} \kappa \hat{\mathfrak{e}} \bar{\gamma}^{K L} \frac{\partial}{\partial \omega_{[\alpha}^{K L}} \frac{\partial}{\partial \omega_{\beta]}^{I J}},
$$

where $\bar{\gamma}^{J}$ are the fiducial flat-space Dirac matrices. Those Clifford-valued operators act on Clifford-valued quantum gravitational precanonical wave functions $\Psi=\Psi\left(\omega_{\alpha}^{I J}, x^{\mu}\right)$ on the total space of the configuration bundle of spin connections over the space-time 国

We can also construct the operator of DW Hamiltonian density $\mathfrak{H}=: \mathfrak{e} H$ restricted to the surface of constraints $C$ given by (4). From (6) and (4) we obtain $\left.(\mathfrak{e} H)\right|_{C}=-\mathfrak{p}_{\omega_{\beta}^{I J}}^{\alpha} \omega_{\alpha}^{I K} \omega_{\beta K}^{J}-\frac{1}{\kappa} \Lambda \mathfrak{e}$ and, using the above representations,

$$
\widehat{H}=\hbar^{2} \varkappa^{2} \kappa \bar{\gamma}^{I J} \omega_{[\alpha}^{K M} \omega_{\beta] M}{ }^{L} \frac{\partial}{\partial \omega_{\alpha}^{I J}} \frac{\partial}{\partial \omega_{\beta}^{K L}}-\frac{1}{\kappa} \Lambda .
$$

\footnotetext{
aWe use the notion of the "bundle of connections" in the sense similar to Ref. 13 rather than e.g. Ref. 14.
} 
Now, the covariant precanonical Schrödinger equation for quantum gravity (cf. (2) )

$$
\mathrm{i} \hbar \varkappa \widehat{\nabla} \Psi=\widehat{H} \Psi,
$$

where $\widehat{\not}:=\hat{\gamma}^{\mu}\left(\partial_{\mu}+\frac{1}{4} \omega_{\mu I J} \bar{\gamma}^{I J}\right)$ and $\hat{\gamma}^{\mu}:=\bar{\gamma}^{I} \hat{e}_{I}^{\mu}=-\mathrm{i} \hbar \varkappa \kappa \bar{\gamma}^{I J} \frac{\partial}{\partial \omega_{\mu}^{I J}}$, can be written in an explicit form (up to an ordering of $\omega$-s and $\partial_{\omega}-\mathrm{s}$ ):

$$
\bar{\gamma}^{I J}\left(\partial_{\mu}+\frac{1}{4} \omega_{\mu K L} \bar{\gamma}^{K L}-\omega_{\mu M}{ }^{K} \omega_{\beta}{ }^{M L} \frac{\partial}{\partial \omega_{\beta}^{K L}}\right) \frac{\partial}{\partial \omega_{\mu}^{I J}} \Psi=-\lambda \Psi,
$$

where $\lambda:=\frac{\Lambda}{(\hbar \kappa \varkappa)^{2}}$ is a dimensionless constant which combines three different scales: cosmological, Planck, and the UV scale $\varkappa$ introduced by precanonical quantization.

The fact that all physical constants have been melted into a single dimensionless constant $\lambda$, which appears as an eigenvalue of the operator in the l.h.s. of eq. (16), suggests that the latter, and the theory of quantum gravity derived from precanonical quantization, may correspond to a statement of purely mathematical nature concerning the sections of the Clifford bundle over the bundle of spin connections over space-time. Surprisingly, eq. (16) is invariant with respect to the scale transformation $x \rightarrow a x, \omega \rightarrow a^{-1} \omega$, so that it knows nothing about the Planck scale, where the effects of quantum gravity are commonly expected to take place. Probably, it is an interaction of gravity with the matter fields, which we neglected here, that (re-)introduces the physical scales.

The scalar product of precanonical wave functions is given by

$$
\langle\Phi \mid \Psi\rangle:=\operatorname{Tr} \int \bar{\Phi} \widehat{[\mathrm{d} \omega]} \Psi, \quad \widehat{[d \omega]}=\mathrm{i}^{\frac{1}{2} n(n+1)-1} \hat{\mathfrak{e}}^{-n(n-1)} \prod_{\mu, I<J} \mathrm{~d} \omega_{\mu}^{I J},
$$

where $\bar{\Psi}:=\bar{\gamma}^{0} \Psi^{\dagger} \bar{\gamma}^{0}$ is the complex conjugate and reverse of $\Psi, \frac{15}{,}$ and $\left.\widehat{[\mathrm{d} \omega}\right]$ with $\hat{\mathfrak{e}}^{-1}=$ $\frac{1}{n !} \epsilon^{I_{1} \ldots I_{n}} \epsilon_{\mu_{1} \ldots \mu_{n}} \hat{e}_{I_{1}}^{\mu_{1}} \ldots \hat{e}_{I_{n}}^{\mu_{n}}$ is a Misner-like $\underline{21}$ diffeomorphism invariant generalizedHermitian (in the sense $\widehat{[\mathrm{d} \omega]}=\widehat{\widehat{[\mathrm{d} \omega]}}$ ) operator-valued measure on the fibers of the bundle of spin connections over space-time. In general, $\operatorname{Tr}(\bar{\Psi} \Psi)$ on a pseudoEuclidean Clifford algebra is not positive-definite. However, on the subspace of functions $\Phi^{ \pm}:=\left(1 \pm \bar{\gamma}^{0}\right) \Psi\left(1 \pm \bar{\gamma}^{0}\right)$, it coincides with the positive definite Frobenius inner product: $\operatorname{Tr}\left(\overline{\Phi^{ \pm}} \Phi^{ \pm}\right)=\operatorname{Tr}\left(\Phi^{ \pm \dagger} \Phi^{ \pm}\right)=:\left\|\Phi^{ \pm}\right\|^{2}$.

The normalizability of precanonical wave functions according to the above scalar product (and quite independently from its details) requires $\hat{\mathfrak{e}}^{-\frac{1}{2} n(n-1)} \Psi$ to vanish at large $\omega$-s. This essentially implies the quantum avoidance of curvature singularities (large $\omega$-s) in the sense that the probability density of observing the regions of space-time with an extremely high curvature vanishes as a consequence of the normalizability of the precanonical wave function of quantum gravity. Note, however, that in spite of its plausibility, this resolution to the singularity problem depends on the actual existence of the properly normalized solutions of eq. (16), which is not yet proven. Moreover, the argument based on the normalizability of precanonical wave functions in its present form ignores interactions with matter, the intricalities related to the indefiniteness of $\operatorname{Tr}(\bar{\Psi} \Psi)$, and the gauge fixing, i.e. the choice of the 
coordinate systems/local orientations of vielbeins on average, which is important for the extraction of the physically relevant information from the solutions of (16).

Eq. (16) can be seen as a variant of a generalized confluent hypergeometric equation of $n$ matrix (Clifford-valued) variables $Z_{\mu}:=\omega_{\mu}^{I J} \bar{\gamma}_{I J}$. To my knowledge, the theory of such equations is not yet sufficiently developed in mathematics, so that even the proof of the existence of properly normalized solutions, which is essential for the present formulation of quantum theory of gravity to be physically viable and mathematically well-defined, is a challenging problem. However, in the regime when the term $\omega \omega \partial_{\omega} \partial_{\omega}$ in (16) can be neglected, we obtain a more tractable equation

$$
\left(\mathrm{i} k_{\mu} \partial_{Z_{\mu}}+\frac{1}{4} Z_{\mu} \partial_{Z_{\mu}}+\lambda\right) \Phi=0,
$$

where $\Psi(x, \omega)=\mathrm{e}^{\mathrm{i} k_{\mu} x^{\mu}} \Phi\left(Z_{\mu}\right)$ and the ordering ambiguity in the $Z \partial_{Z}$ term is accounted for in a re-definition of $\lambda$. By separating the variables $Z_{\mu}$, so that $\Phi(Z)=\Pi_{\mu} \Phi\left(Z_{\mu}\right)$ and $\lambda=\Sigma_{\mu} c_{\mu}$, we obtain the following equation for each $\mu$ :

$$
\left(\mathrm{i} k \partial_{Z}+\frac{1}{4} Z \partial_{Z}\right) \Phi+c \Phi=0 .
$$

Its solution (written in terms of the formal fractional power of the matrix in the square brackets $\underline{17})$ i.

$$
\Phi \sim[4 \mathrm{i} k+Z]^{-4 c} .
$$

The corresponding probability density of spin connections given by $\|\Phi\|^{2}$ behaves as $\sim\|Z\|^{-8 c}$ at $|k| \ll\|Z\|$, and its normalizability with the measure in (17) restricts the admissible values of $c$. The above heavy-tailed power law asymptotic behavior is characteristic of Lévy processes, with the exponent known to be related to the fractal dimensionality of the trajectories of Lévy flights $\frac{18}{}$ in the target spaces, which are fibers of the bundle of spin connections here. A Lévy flight on each fiber of the bundle means a random spin connection field over space-time with a generally non-Gaussian distribution defined by the precanonical wave function. The properties of this omnipresent random field will be reflected in the properties of test particles propagating in space-time, their trajectories, spatio-temporal distribution and correlations, which can be potentially observable. The fractality of the Lévy flights in the target space of spin connections may be translated to the effective fractality of space-time itself as one of the consequences of quantum gravity. We hope to explore the details of that in the future.

In the opposite regime, when the term $\omega \omega \partial_{\omega} \partial_{\omega}$ in (16) dominates (and the wave numbers $k_{\mu}$ are not too high), eq. (16) reduces to a matrix generalization of a simple PDE: $u^{2} \Phi^{\prime \prime}(u)-\lambda \Phi(u)=0$, whose power law solutions $\Phi \sim u^{\frac{1}{2}(1 \pm \sqrt{4 \lambda+1})}$ again point to a restriction on the admissible values of the cosmological constant in $\lambda$ from the normalizability of the wave function at large $\omega$-s, and to a heavy-tailed

\footnotetext{
${ }^{\mathrm{b}}$ A solution similar to (20) was obtained also in the cosmological context $\underline{10} c, \underline{16}$, where the $\omega \omega \partial_{\omega} \partial_{\omega}$ term in (16) vanishes identically because of the cosmological symmetries of the problem, which leave non-vanishing only one independent component of the spin connection.
} 
Lévy-type behavior at large $\omega$-s, albeit with a different fractal dimensionality of the Lévy flights in this regime.

The Green functions of (16) are transition amplitudes between the values of spin connections at different points: $\left\langle\omega, x \mid \omega^{\prime}, x^{\prime}\right\rangle$. They describe a quantum space-time geometry which generalizes the classical geometry formulated in terms of smooth spin connection fields $\omega(x)$. By noticing an analogy with the statistical hydrodynamics approach to turbulence, $\underline{19}$ which replaces the description in terms of the smooth velocity fields $\mathbf{v}(\mathbf{x})$ with the statistical description in terms of the multipoint velocity correlators, we can speak of the picture of quantum gravity derived from precanonical quantization as a space-time turbulence or, in the mathematical context of the present formulation, a spin connection foam. In fact, this picture is even closer to the original Wheeler's intuition about the "space-time foam" than his quantum geometrodynamics based on the Wheeler-De Witt equation and the notion of an infinite-dimensional superspace of all metrics.

Note also that the metric tensor in the present formulation is an operator:

$$
\widehat{g}^{\mu \nu}=-\hbar^{2} \varkappa^{2} \kappa^{2} \eta^{I J} \eta^{K L} \frac{\partial^{2}}{\partial \omega_{\mu}^{I K} \partial \omega_{\nu}^{J L}} .
$$

Hence, the intervals $\widehat{d s}^{2}=\widehat{g}_{\mu \nu} d x^{\mu} d x^{\nu}$ are operator-valued. This makes the notion of the distances between points, and hence the operational notion of a point of the physical space-time, fuzzy due to the quantum nature of the space-time geometry. In this way the description of quantum geometry of space-time derived from the precanonical quantization of general relativity complements the ideas about quantum space-time being discussed within the framework of LQG, string theory and non-commutative geometry.

\section{Possible connections with LQG}

A first discussion of the constraints in the Ashtekar formulation using a version of the DW (multisymplectic) Hamiltonian theory applied to the vielbein EinsteinPalatini Lagrangian (3) can be found in the paper by Esposito e.a. 20

In spite of the obvious differences of our precanonical approach from the LQG programme, which (i) uses a $(3+1)$-splitting vs. our explicitly space-time symmetric approach, (ii) heavily relies on the specifics of four-dimensional globally hyperbolic space-times vs. our intention to develop a formalism potentially applicable in any number of dimensions and any signature of space-time, (iii) uses functionals and functional derivative operators vs. our use of functions and partial derivative operators, etc., there are some striking similarities as well, one of them being the emergence, after Hamiltonization, of a formulation based on the connections, with the vielbeins, or the densitized inverse triads/dreibeins in the Ashtekar formulation, represented as differential operators with respect to the connections.

Whereas our approach to quantization of gravity is based on the fundamental brackets in the Weyl subalgebra in the subspace of Hamiltonian 0 - and $(n-1)$-forms, 
our construction of the Poisson-Gerstenhaber brackets in field theory $\underline{2}$ also offers another, yet unexplored opportunity based on the Dirac bracket between the spin connection 1-form $\omega_{\alpha}^{I J} d x^{\alpha}$ and its conjugate $(n-2)$-form of polymomenta: $\mathfrak{p}_{\omega_{\nu}^{K L}}^{\mu} \varpi_{\mu \nu}$, where $\varpi_{\mu \nu}:=\imath_{\partial_{\mu}} \iota_{\partial_{\nu}} \varpi$ :

$$
\left\{\omega_{\alpha}^{I J} d x^{\alpha}, \mathfrak{p}_{\omega_{\nu}^{K L}}^{\mu} \varpi_{\mu \nu}\right\}^{\mathrm{D}}=\left\{\omega_{\alpha}^{I J} d x^{\alpha}, \mathfrak{p}_{\omega_{\nu}^{K L}}^{\mu} \varpi_{\mu \nu}\right\} \sim n \delta_{K}^{[I} \delta_{L}^{J]} .
$$

This bracket is a gravitational analogue of the bracket between the potential 1form $\mathrm{A}=A_{\mu} d x^{\mu}$ and the $(n-2)$-form of the dual field strength in Electrodynamics, $* \mathrm{~F}=* \frac{1}{2} F_{\mu \nu} d x^{\mu} \wedge d x^{\nu}$, found in Ref. 2 (see also Refs. 22, 23). After a (3+1)-splitting, a restriction to the initial data surfaces and integration over them (cf. Refs. 2, 22), the bracket can be related to the fundamental Poisson bracket underlying the Ashtekar formulation in (3+1)-dimensions, and the forms involved in (21) can be used to construct the holonomy-flux variables underlying the LQG approach (see e.g. Refs. 24 for a review). However, unlike the subalgebra of 0 - and ( $n-1)$-forms in (77)-(12), the set of $(n-2)$ - and 1-forms is not closed with respect to the PoissonGerstenhaber bracket operation, hence the closure of the subalgebra involves forms of all the degrees $\leqslant(n-2)$.

A further understanding of possible connections of precanonical quantization with LQG in $(3+1)$ dimensions requires an inclusion of the Holst term $\underline{25}$ in the Einstein-Palatini Lagrangian (3) in order to incorporate the Barbero-Immirzi parameter, that does not, however, appear to be necessary within our approach. Interestingly, this property is shared with a multidimensional generalization of the LQG-type connection formulation of pure gravity discussed by Bodendorfer e.a. $\underline{26}$ Hence, it is likely that more connections can be found between that formulation and the precanonical approach of the present paper.

\section{References}

1. H. Rund, The Hamilton-Jacobi Theory in the Calculus of Variations, (Robert E. Krieger, N.Y., 1973); H. Kastrup, Phys. Rep. 101, 1 (1983).

2. I. Kanatchikov, arXiv:hep-th/9312162;

I. Kanatchikov, Rep. Math. Phys. 41, 49 (1998). arXiv:hep-th/9709229

I. Kanatchikov, Rep. Math. Phys. 40, 225 (1997). arXiv:hep-th/9710069.

3. I. Kanatchikov, Int. J. Theor. Phys. 37, 333 (1998). arXiv:quant-ph/9712058.

I. Kanatchikov, AIP Conf. Proc. 453, 356 (1998). arXiv:hep-th/9811016.

I. Kanatchikov, Rep. Math. Phys. 43, 157 (1999). arXiv:hep-th/9810165.

4. I. Kanatchikov, arXiv:hep-th/0112263; I. Kanatchikov, in The Ninth Marcel Grossmann Meeting, p. 1395, (World Scientific, Singapore, 2002). arXiv:gr-qc/0012038.

5. M. de León, M. Salgado and S. Vilariño, arXiv:1409.5604

6. I. Kanatchikov, J. Geom. Symmetry Phys. 37, 43 (2015). arXiv:1501.00480.

7. I. Kanatchikov, to appear in MG14 Proceedings (2016). arXiv:1602.01083.

8. I. Kanatchikov, Adv. Theor. Math. Phys. 18, 1249 (2014). arXiv:1112.5801. 
I. Kanatchikov, arXiv:1312.4518.

9. A. Slavík, Product Integration, its History and Applications, (Matfyzpress, Praha, 2007).

10. I. Kanatchikov, AIP Conf. Proc. 1514, 73 (2012). arXiv:1212.6963.

I. Kanatchikov, J. Phys. Conf. Ser. 442, 012041 (2013). arXiv:1302.2610,

I. Kanatchikov, NPCS 17, 372 (2014). arXiv:1407.3101.

I. Kanatchikov, Precanonical Quantization: From Foundations to Quantum Gravity, in preparation.

11. I. Kanatchikov, Int. J. Theor. Phys. 40, 1121 (2001). arXiv:gr-qc/0012074. See also: I. Kanatchikov, gr-qc/9810076; gr-qc/9912094; gr-qc/0004066.

12. I. Kanatchikov, in Differential Geometry and its Applications, (World Scientific, Singapore, 2008). arXiv:0807.3127.

13. M. Castrillón López and J. Muñoz Masqué, Math. Z. 236, 797 (2001).

14. K. Mitter and C.-M. Viallet, Commun. Math. Phys. 79, 457 (1981).

15. J. Snygg, Clifford Algebra. A Computational Tool for Physicists, (Oxford Univ. Press, N.Y., 1997). (Our $\bar{\Psi}$ is Snygg's $\Psi^{\dagger}$ on p. 171 , our $\Psi^{\dagger}$ is the Hermitian adjoint of $\Psi$.)

16. I. Kanatchikov, Precanonical Quantum Cosmology and Cosmological Spin Connection Field, talk at MG14 (2015).

17. N.J. Higham, Functions of Matrices, theory and computation, (Philadelphia, SIAM, 2008).

18. V. Zaburdaev, S. Denisov and J. Klafter, Rev. Mod. Phys. 87, 483 (2015).

19. M.I. Vishik and A.V. Fursikov, Mathematical Problems of Statistical Hydromechanics, (Kluwer, Dordrecht, 1988)

20. G. Esposito, C. Stornaiolo and G. Gionti, Nuovo Cim. B110, 1137 (1995). arXiv:gr-qc/9506008.

21. C. Misner, Rev. Mod. Phys. 29, 497 (1957).

22. F. Hélein and J. Kouneiher, Adv. Theor. Math. Phys. 8, 735 (2004). arXiv:math-ph/0401047.

23. Y. Kaminaga, EJTP 9, 199 (2012);

S. Nakajima, arXiv:1510.09048,

24. Dah-Wei Chiou, arXiv:1412.4362,

K. Banerjee, G. Calcagni and M. Martin-Benito, SIGMA 8, 016 (2012). arXiv:1109.6801.

25. S. Holst, Phys. Rev. D53, 5966 (1996). arXiv: gr-qc/9511026.

26. N. Bodendorfer, T. Thiemann and A. Thurn, CQG 30, 045001, 045002, 045003, 045004 (2013). arXiv:1105.3703, arXiv:1105.3704, arXiv:1105.3705, arXiv:1105.3706. 